\title{
Die Torlak, eine Volksgruppe zwischen Christentum und Islam
}

\author{
IRENE BeLDicEANU-STEINHERR
}

Das abenteuerliche Leben des Schejhs Bedreddin und der damit verbundene Aufstand in Westanatolien haben nicht nur in den frühen osmanischen Chroniken ihren Niederschlag gefunden, sondern auch das Interesse der Historiker moderner Zeiten erweckt, so dass wir über zahlreiche Studien verfügen, die allerdings manche Fragen offen lassen. ${ }^{1}$ Der Beitrag befasst sich nur mit einem Aspekt dieser Ereignisse, dem Auftauchen einer „Torlak“ genannten Gruppe. Unser Wissen auf diesem Gebiet konnte bis jetzt nicht viel mehr über das hinausgehen, was der Bericht vom Aufstand uns bisher geboten hatte. Die ErschlieBung osmanischer Steuerregister, die Bekanntmachung osmanischer Heiligenlegenden sowie die neuen technischen Errungenschaften wie das Internet haben inzwischen neue Forschungen mit neuen Ergebnissen ermöglicht.

Die Beschäftigung mit Kleinasien brachte überraschenderweise bis jetzt unbekanntes Material über diese Volksgruppe zu Tage. Ein zufälliger Blick ins Internet fuihrte ebenfalls zu einer neuen Perspektive, denn da war nicht die Rede von Anatolien, sondern nur vom Balkan. Plötzlich erschien diese Volksgruppe wie der Gott Janus mit zwei Gesichtern. Das eine war nach Europa gerichtet, das andere nach Kleinasien. Somit erwies sich dann auch, dass bei den Historikern diese Spaltung ebenfalls bemerkbar ist. Wer den Balkan kennt, ignoriert Kleinasien - und umgekehrt, was natülich der Schwierigkeit zuzuschreiben ist, die Sprachen der verschiedenen Regionen zu beherrschen.

Forscher, die ihr Interesse dem Balkan gewidmet haben, kennen nur die Torlak, die in einem Gebiet wohnen, das sich auf den Süden Serbiens sowie Bulgarien, Mazedonien, auf den Kosovo und einen Teil Rumäniens erstreckt. Die meisten Torlak auf dem Balkan sprechen einen slawischen Dialekt, den man Torlakian nennt. Die Mehrheit ist orthodox. Einige Gruppen sind Muslime und eine Minderheit Katholiken. Diese Einzelheiten verdanken wir einem Mönch Kiril Zhivkovich (Živković) aus Pirot. ${ }^{2}$ Er verfasste 1762 ein Werk sprachwissen-

1 Anlässlich der Gedenkfeier für die 2009 verstorbene Frau Prof. Irène Mélikoff wurde ein gekürzter Vortrag ohne Quellenangabe am 20. Januar 2010 an der Universität Marc Bloch in Straßburg gehalten und zwar im Rahmen der Abteilung für persische und türkische Studien unter der Leitung von Professor Paul Dumont.

2 Internet-Google.fr (23. Oktober 2010),Stichwort Torlak (unter Torlakian Dialect): $<$ http://en.wikipedia.org/wiki/Torlakian_dialect>; Manuscript aus dem Temska Klo- 
schaftlicher Natur, dem er diese Angaben einverleibt hat. Serbische Enzyklopädien und Wörterbücher geben uns ebenfalls Auskunft über diese Volksgruppe. ${ }^{3}$

Forscher auf dem Gebiet der Osmanistik ignorieren die Torlak auf dem Balkan, und ich muss gestehen, dass ich auch zu diesen gehörte. Eine türkische Kollegin machte mich darauf aufmerksam, dass die osmanischen Steuerregister, die den Balkan betreffen, ebenfalls Material über die Torlak enthalten. ${ }^{4}$ Ich selber hatte nicht im Geringsten an diese Möglichkeit gedacht, obwohl die vom Archiv gedruckten Ausgaben bei mir in der Bibliothek stehen!

Der Beitrag besteht aus drei Teilen, wobei er sich mit einer Ausnahme auf osmanische Quellen beschränkt. Der erste Teil behandelt die Torlak auf europäischem Boden, der zweite Teil die Geschehnisse, die sich in Kleinasien abgespielt haben. Der dritte Teil soll einige Überlegungen über den eventuellen Ursprung dieser Volksgruppe enthalten. Er endet mit einer Zusammenfassung der neuen Erkenntnisse.

\section{Die Torlak auf europäischem Boden}

\section{a. Steuerregister}

Als Erstes sind zwei Steuerregister zu erwähnen. Es handelt sich um das Register TD 370 aus dem Jahre 1530. ${ }^{5}$ Dort ist die Rede von einem Dorf namens Torlak im Bezirk von Tutrakan. Die Einwohner, alles Ungläubige, sind mit der

ster in Serbien, verfasst vom Mönch Kiril Živković aus Pirot; Stichwort „Kiril Zhivkovich“, Dialecte de Torlak.

3 Siehe Artikel „Torlak“ in Karadžić, 1852, 744, und Milenović 1929, 576; Kurze Notiz zu ,Torlak“ in Mala Enciklopedija, ${ }^{2} 1969,715$ (freundliche Mitteilung von Saša Popović).

4 Ich verdanke diese Auskunft Ayşe Kayapınar aus Ankara, die mir eine Fotokopie von den Texten zugeschickt hat. Ihre Kenntnisse des Türkischen und Bulgarischen gaben ihr die Möglichkeit sich auf die Register des Balkans zu spezialisieren.

5 Prof. Machiel Kiel hat der Gegend von Razgrad in der Festschrift Irène Mélikoff einen Artikel gewidmet unter dem Titel „Hrāzgrad-Hezārgrad-Razgrad. The Vicissitudes of a Turkish Town in Bulgaria“, siehe Kiel 1991, 495-563. Zu seiner Zeit war das Register TD 370 (Tapu defteri $\mathrm{n}^{\circ}$ 370) aus dem Archiv des Ministerpräsidialamtes in Istanbul noch nicht veröffentlicht. Inzwischen verfügen wir über den Druck: 370 numaral Muhâsebe-i Vilâyet-i Rûm-Ili Defteri (937/1530), Bd.I, 2001; Bd. II, 2002. Prof. Kiel datiert das Register auf das Jahr 1516. Man darf nicht vergessen, dass Süleyman der Prächtige eine Zusammenstellung der Einkünfte des Reiches befohlen hatte, worauf eine ganze Reihe von Kurzfassungen erstellt wurde, die sich auf die letzte Landaufnahme stützte. Deshalb besteht kein Grund das Jahr 1516 für das ausführliche Register anzuzweifeln. Prof. Kiel teilte mir mit, dass das Dorf 1934 in Zar Kaloyan umgetauft wurde. Ab 1951 erhielt es den Namen Hlebarovo. Inzwischen ist vielleicht wieder eine Änderung eingetreten. 
Instandhaltung der Festung „Cernovi“ beauftragt. ${ }^{6}$ Das Dorf wird in einem anderen Register zitiert, das TD 382 aus demselben Archiv, ebenfalls aus der Zeit Süleymans des Prächtigen; es trägt allerdings kein Datum. ${ }^{7}$ Das Dorf gehörte immer noch zur Domäne Ibrahim Paschas. Die Einwohner bewachten seit geraumer Zeit die Pässe, betont der Text. Deshalb gewährte ihnen der Staat eine Befreiung von mehreren Steuern (S. 244-246). Alle Einwohner haben christliche Namen slawischen Ursprungs. Eine einzige Person nennt sich Ibrahim Sohn des Abdullah. Das bedeutet, dass er ursprünglich ein Christ war und später zum Islam übergetreten ist.

\section{b. Eine Heiligenlegende}

Eine weitere osmanische Quelle ist die Heiligenlegende des Demir Baba, dessen Grabmal sich im heutigen Bulgarien in der Nähe von Razgrad befindet. Die Gegend ist in den osmanischen Quellen als Deliorman [Verrückter Wald] oder Ağaç Denizi [Baummeer] bekannt. Die Legende selbst, die von Bedri Noyan gedruckt worden ist, ist leider schwer verfügbar, und Riza Yıldırım, der sie benutzt hat, bedauert die unbefriedigende Veröffentlichung. ${ }^{8}$ Immerhin müssen wir dankbar sein, dass er uns einen Teil des Inhalts wiedergibt. Die Legende beschreibt den zweimaligen Besuch Demir Babas im Kloster von Seyyid Ali Sultan bei Didymoteichon, dem osmanischen Dimetoka. Demir Baba muss nach Schätzung Rıza Yıldırıms um die Mitte des 16. Jahrhunderts gelebt haben. Sein Kloster, auf Türkisch Tekke, befindet sich in Bulgarien in der Nähe von Razgrad. ${ }^{9}$ Als Demir Baba in das Gebiet kam, wo die Nachkommen Seyyid Ali Sultans wohnten, gab es dort nicht eines, sondern zwei Klöster: ein unteres und ein oberes. Zwischen den Bewohnern der beiden Klöster herrschte Unfrieden. Die Bewohner des unteren Klosters beschimpften die Bewohner des oberen Klosters, die in der Legende immer als Bektaschi und Torlak bezeichnet werden. Sie nannten sie grindige Juden (keleş s fit Mensch), mit Zaumzeug versehener Jude (mülcem çıfıt) oder jüdischer Zimmer-

6 TD 370, 556. Das Dorf gehörte zu den Domänen Ibrahim Paschas.

7 Das Register soll kurz vor 1551 entstanden sein: M. Kiel, op. cit., 539.

8 Yildirım 2010, 178, Anm. 43.

9 Babinger, der das Kloster im Jahr 1930 besucht hat, gibt uns eine kurze Beschreibung begleitet von einer Karte in: Babinger 1962, 88-96. Eine Zeichnung des Kloster von Felix Philipp Kanitz (1829-1904) sowie weitere Bilder vom Kloster befinden sich im Internet (Datum 24. Okt. 2010), Stichwort Demir Baba (images correspondant à Demir Baba): <http://en.wikipedia.org/wiki/Demir_Baba_Teke>. Das Thema ist mehrmals behandelt worden. 
mann (naccar çı fit)..$^{10}$ Die oberen verwendeten nicht weniger beleidigende Ausdrücke für die unteren Klosterbrüder, wie unsaubere oder ungläubige Türken. Sie wollten von Demir Baba nichts wissen, bedrohten ihn und nannten ihn ein türkisches Schwein (donuz [domuz] türk). ${ }^{11}$ Im oberen Kloster wohnten mit der schriftlichen Erlaubnis der Bektaschis zwei Burschen, die Janitscharen waren. Die unteren Klosterbrüder nannten sie Zigeunerjungen oder Judenburschen. ${ }^{12}$ Sie werden als blau- oder grünäugig, mit einem um die Taille geschlungenen „fitsen“"13 beschrieben (beline fitsen gìmiş), mit einer Bektaschimütze auf dem Kopf und einem bläulichen Gesicht. Aus diesem Bericht geht hervor, dass die zwei Jugendlichen, die in dem Kloster wohnten, ursprünglich keine Muslime waren.

\section{c. Europäische Quellen}

Eine Beschreibung der Torlak findet man auch in drei im Westen veröffentlichten Werken ohne Anspielung auf politische Ereignisse. Dies sind die Werke von Menavino ${ }^{14}$, Spandugino ${ }^{15}$ und Nicolas de Nicolay. ${ }^{16}$ Bei den zwei letzteren handele es sich um eine Erweiterung Menavinos mittels anderer Quellen, wie es die Herausgeber des Textes von Nicolas de Nicolay beweisen. Der Grundtext dürfte der des Menavino sein, weil er im Jahre 1504 im Alter von zwölf Jahren von Piraten gefangen wurde und zehn Jahre am Hofe des Sultans verbracht hat.

Menavino widmet einige Kapitel religiösen Gemeinden. Seine Beschreibung der Torlak ist nicht schmeichelhaft. Sie sind mit einem Schafsfell bekleidet. Sie rasieren ihren Schädel und brennen ihre Schläfen mit einem Stück Baumwolle. Sie können nicht lesen, leben von Almosen und betätigen sich als Handleser und

10 Yıldırım, op. cit., 180. Es ist wahrscheinlich eine Anspielung auf Torlak Hu Kemal, der in Anatolien in der Gegend von Manisa einen Aufstand angezettelt hat und von dem man berichtet, er sei ein zum Islam übergetretener Jude gewesen.

11 Ebd., op. cit., 180.

12 Ebd., op. cit., 183.

13 Der Verfasser des Artikels konnte keine Erklärung über dieses unbekannte Wort geben.

14 Menavino 1548, Trattato de'costumi et vita de'turchi, Kapitel XIII, 79-82. (Zugang zum Text durch Internet: <http.//books.google.fr $>$ )

15 Théodore Spandugino: Petit traicté de Théodore... Cantacusin [Contacuzène], Constantinopolitain, de l' Origine des princes ou empereurs des Turcz, ordre de leur court et coustumes de la nation, Manuskript $\mathrm{n}^{\circ}$ 797, Bibliothèque du Château de Chantilly, Manuskript 797.

(Musée de Condé in Chantilly, alte $\mathrm{N}^{\circ}$ XIV H 97) fol. $49 \mathrm{r}^{\circ}-50^{\circ}$; in: URL: <http:// www.calames.abes.fr/pub/\#details?id=IF3011295\%23culture $=$ fr $>$.

Ein Verzeichnis der Kapitel befindet sich in Villain-Gandossi 1979, 155-156, Anmerkung 27. Ich verdanke der Autorin die Kopie des Abschnittes über die Torlak.

(Übersetzung ins Englische: Spandounes 1997, 138-139).

16 Nicolay 1989, 194-197 (mit einer Zeichnung 196). 
Wahrsager. Sie spotten über die Leichtgläubigkeit der Menschen, die es ihnen erlaubt, sich satt zu essen. Sie nehmen Drogen zu sich, haben ein sittenloses Verhalten und benehmen sich wie die wilden Tiere.

\section{Die Torlak in Kleinasien}

a. Die Vita des Schejchs Bedreddin und die Aufstände in Kleinasien

In den islamischen Quellen und in der Vita des Schejhs Bedreddin spricht man nur von dieser Volksgruppe im Zusammenhang mit dem abenteuerlichen Leben des Schejchs und den Personen, die als seine Anhänger betrachtet werden. Manche bestreiten allerdings den Kontakt mit Bedreddin, und zwar mehr aus politischen Gründen. Man wollte die Familie nicht mit den zum Tode verurteilten Anhängern in Beziehung bringen.

Nach der Lebensbeschreibung des Enkels ${ }^{17}$ stammte Bedreddin aus einer Ortschaft in Thrakien, auf Türkisch Samavna genannt. ${ }^{18}$ Sein Vater behauptete, ein Abkömmlung der seldschukischen Sultane zu sein. Seine Mutter war eine Christin. Schon in jungen Jahren wurde er in die Wissenschaft des Islams eingeweiht. Eines Tages verlässt er Thrakien und begibt sich auf eine lange Reise, die ihn unter anderem auch nach Ägypten führt. Er begegnet in Kairo Hüseyin aus Ahlat, einer Stadt am Nordufer des Vansees, und wendet sich von diesem Augenblick an vom Studium des klassischen Islams ab. Er begeht den Weg eines extremen Sufismus mit ausgedehnten bis zur Bewusstlosigkeit geführten Fastenperioden. Nach dem Tod seines Meisters beschließt er, nach Thrakien zurückzukehren. Er kommt zuerst ins Land von Aydın, wo seine Predigten viel Erfolg haben. Bei dieser Gelegenheit besucht er auch die Insel Chios und das dortige Kloster und wird von den Mönchen freundlich empfangen. Die Reise führt ihn anschließend über Kütahya nach dem Pass von Domaniç, ursprünglich die Sommerweide der ersten Osmanenherrscher. Er erreicht Serme im Osten von Bursa $^{19}$, wo ihn die Bevölkerung begrüßt. Es handele sich um Torlak, betont der

17 Halil bin Ismâ̂1 bin Şeyh Bedrüddîn Mahmûd 1967. Abdülbâki Gölpınarlı hatte ein Jahr zuvor eine Studie Simavna Kadısıoğlu Şeyh Bedreddin veröffentlicht, (Gölpınarlı 1966). Eine ausführliche Inhaltsangabe auf Deutsch begleitet von einer mit vielen Anmerkungen versehenen Studie verdanken wir H. J. Kissling, siehe Kissling 1950.

18 Über den Ort: Soustal 1991, 175, Ammobunon mit Verweis auf verschiedene türkische Formen.

19 Serme: Karte der Türkei, Ankara, Direktion für Kartographie, Maßstab 1/200 000, Blatt Bursa, t/28. Die Route beweist, dass Bedreddin einen großen Umweg gemacht hat, um von der Provinz Aydın nach Edirne zu kommen. Die älteste Erwähnung von Serme in osmanischen Steuerregistern: Archiv des Ministerpräsidialamtes in Istanbul, Register 
Text. Bedreddin lässt sich mit den Torlak auf eine lange Diskussion ein. Diese sind von seiner außerordentlichen persönlichen Ausstrahlung beeindruckt. Sie begleiten ihn bis vor die Tore der Stadt Bursa. Von dort aus erreicht er Adrianopel.

Als es Musa, dem Sohn Bayezids I., gelang, seinen Bruder Süleyman, genannt Emir Süleyman, am 17. Februar 1411 zu beseitigen, ernannte er Bedreddin zum Heeresrichter (Kadiasker). Zwei Jahre später räumte Mehmed, ebenfalls ein Sohn Bayezids I., seinen Bruder Musa aus dem Weg (5. Mai 1413) und regierte fortan als Sultan Mehmed I. über das ganze osmanische Territorium in Kleinasien wie auch in Europa. Er verbannte Bedreddin nach Iznik (Nikaia) und gewährte ihm eine Rente.

In diesem Augenblick brechen in Anatolien im Land von Aydin und Saruhan Aufstände aus. Der erste findet in der Gegend von Karaburun statt, eine Landschaft am östlichen Ufer des Golfes von Izmir (Smyrna), unweit der Insel Chios. Der Anstifter war Börklüce Mustafa. Er wird als Vertrauter Bedreddins angesehen. Die Verbindung zwischen den beiden Personen wird von der Vita Bedreddins bestätigt. Der zweite Aufstand fand in Manisa statt, angeführt von einer Person, die sich Torlak Hu Kemal nannte. ${ }^{20}$ Neşrî, der Verfasser einer osmanischen Chronik, berichtet, dass er der Chef von mehreren hundert Torlak war, was plausibel ist, wenn man an die bitteren Kämpfe gegen die Truppen des Sultans denkt. Beide Aufstände wurden unterdrückt und die Urheber hingerichtet. Bedreddin war inzwischen nach Bulgarien in die Deliorman genannte Region geflüchtet. Er wurde gefangen genommen und im Dezember 1416 in Serres gehängt.

Die vom Enkel Bedreddins verfasste Lebensgeschichte des Großvaters bewahrt einen gewissen Abstand zu den Geschehnissen in Anatolien, vermutlich, weil die Familie nicht kompromitiert werden wollte. Eine ausführliche Beschreibung der Aufstände findet man in mehreren Chroniken, zum Beispiel bei Âșıkpaşaoğlu ${ }^{21}$ und Neşrîn ${ }^{22}$ sowie bei Doukas ${ }^{23}$, die alle drei die Aufständigen mit Verachtung erwähnen. Diese Ereignisse sind dann mehrmals behandelt worden. $^{24}$

TD 23, 1487, fol.19v (40). Die christliche Bevölkerung von Serme war mit der Pflege der staatlichen Wasserbüffel beauftragt.

20 Die Chroniken sind sich über den Namen nicht einig.

21 Kreutel 1959; Âșıkpaşaoğlu Ahmed Âșıkî 1947.

22 Mehmed Neşri 1995, 543-547 (Text in arabischen Buchstaben und Umschrift).

23 Ducas 1958, 148-152; englische Übersetzung: Ducas 1975, 119-121; französische Übersetzung: J. Dayantis, Ducas 2008.

24 Babinger, op. cit, Anm. 1 und 2; Babinger 1921, 1-106; eine ausführliche Studie von H. J. Kissling, siehe Anm. 17. H. I. Cotsonis 1957, (Bibliographie 397). Weitere Bibliogra- 
Die Vorwürfe, die man den Aufständischen machte, beruhten auf der Meinung, dass sie eine neue soziale Struktur anstrebten, eine Mischung von Christentum und Islam. Diese Haltung konnte weder von den orthodoxen Byzantinern noch von den strengen Muslimen gebilligt werden. Heterodoxe Muslime sehen heute diese Bewegung als einen Ausdruck des Alevismus an, wie ein Blick ins Internet vermuten lässt. Christlicher Einfluss war unvermeidbar, da Bedreddins Mutter, Frau und Schwiegertochter Christinnen waren. Hinter religiösen Rechtfertigungen liegen aber oft verschwiegene Gründe.

Bei der Schlacht von Ankara (1402) wurde Bayezid I. von den Streitkräften der anatolischen Emire im Stich gelassen. Nur die Janitscharen blieben ihm treu. Als Mehmed I. das Zepter ergriff, muss er gegen die untreuen Timarioten ${ }^{25}$ eine Säuberungsaktion vorgenommen haben. Das bezeugt ein von H. Inalcık veröffentlichtes Steuerregister. Die Strafe bestand aus einer Verbannung (sürgün) nach Albanien. ${ }^{26}$ Ein weiterer Beweis bietet eines der ältesten Steuerregister vom Landkreis Aydın. Bei einer Reihe von Timaren verzeichnet es, dass es sich zur Zeit des Sultans in den Händen von dieser oder jener Person befand, worauf sich das Wort Sultan auf Mehmed I. bezieht. Die Nennung des Herrschers nur als Sultan ohne Namen ist bezeichnend für dieses Register. Hinzu kommt, dass die Aufzählung der Herrscher, die die Timare vergeben haben, immer mit Mehmed I. anfängt. Bayezid I., sein Vater wird erst nach ihm genannt, was ungewöhnlich ist. ${ }^{27}$ Bedenkt man, dass Torlak Hu Kemal über mehrere hundert Kämpfer verfügte, dann darf man vermuten, dass er der Chef einer Militäreinheit war, die sehr wahrscheinlich ebenfalls von den Maßnahmen Mehmeds I. betroffen wurde. Es kann kein Zufall sein, dass der Aufstand kurz nach der Machtübernahme Mehmeds I. ausgebrochen ist.

phie auch mit Übersetzungen ins Türkische im Internet. Die letzte ausführliche Studie: Balivet 1995.

25 Besitzer eines Timars, auf türkisch ,timar eri“. Der Staat überließ einen Teil der Steuern, die die Dorfbewohner dem Staate schuldig waren, einer Person aus dem Rang der Sipahi. Diese war verpflichtet in den Krieg zu ziehen mit einer militärischen Ausrüstung, die nach der Höhe der Einkünfte berechnet wurde.

26 Inalcik 1954, Einleitung XXXVI, Anm. 249. Genannt sind die Gebiete von Kocaeli, Eflugan, Canik und Vize, die man im Namensverzeichnis auffinden kann. Mit Ausnahme von Vize handelt es sich um Gebiete in Anatolien. Von Kocaeli wissen wir, dass nach der Schlacht von Ankara ein Teil von Kocaeli den Byzantinern überlassen wurde. Die Verbannung war eine Bestrafung der Verräter.

27 Register des Landkreises Aydın: Istanbul, Archiv des Ministerpräsidialamtes, Fonds Maliyeden Müdevver (MAD) 232, aus dem Jahr 1467, sowie MAD 16027, das ein Fragment desselben Registers ist. 


\section{b. Ein osmanisches Steuerregister}

Vor ein paar Jahren stieß ich ganz unverhofft auf eine Stelle in einem Steuerregister, in dem von vier von Torlak bewohnten Dörfern die Rede ist. ${ }^{28}$ Leider gibt uns das Register den Namen der Dörfer nicht bekannt. Es datiert von Anfang 871 der Hidschra, was dem 13. August des Jahres 1466 entspricht. Wir haben es mit der zweiten Landaufnahme der Regierung Mehmeds II. zu tun. Das Register ist den Infanteristen (auf Türkisch yaya oder auf Persisch piyadegan) des Bezirkes von Sultanöyügü gewidmet. Das Zentrum war Eskişehir. Der Text befasst sich mit den Infanteristen der Unterabteilung von Sivrihisar, die mit dem Bezirk von Ankara eine gemeinsame Grenze hatte. Hier die Übersetzung:

Ibrahim, der Sohn des Zekeriya, der zu den Infanteristen des Bezirkes von Ankara gehörte, war mit anderen Infanteristen aus vier von Torlak bewohnten Dörfern verschwunden. Er wurde gefunden und wieder als Infanterist eingesetzt.

Infanteristen bekamen vom Staat ein Stück Ackerland. Als Gegenleistung mussten sie in den Krieg ziehen. Register dieser Art beweisen den großen Verlust an Menschen, die, wie wir heute sagen, als Kanonenfutter dienten. Dass manche diesem Schicksal durch Flucht entgehen wollten, ist nicht verwunderlich. ${ }^{29}$

Trotz der großen Menge von Steuerregistern, die ich bis jetzt eingesehen habe, konnte ich in den Bezirken des restlichen Kleinasiens keine weiteren Torlak finden.

\section{Welche Schlussfolgerungen können wir aus dem hier erwähnten Material ziehen?}

\section{a. Religion und sozialer Stand der Torlak}

Als erstes ist auffällig, dass die Torlak nicht alle derselben Religion angehörten. Auf dem Balkan waren es meistens Christen; es gab aber auch Muslime, sicher spätere Konvertiten, und auch eine geringe Anzahl von Katholiken. Man muss allerdings bedenken, dass die Quelle aus der zweiten Hälfte des 18. Jahrhunderts stammt. Ein Steuerregister erlaubt die Anfänge einer Islamisation der Torlak auf

28 Archiv des Ministerpräsidialamtes in Istanbul, Abteilung MAD n ${ }^{\circ}$, fol.8r ${ }^{\circ}$.

29 Die Verstorbenen wurden am Rande des Registers eingetragen und sind fast auf jeder Seite zitiert: Istanbul, Archiv des Ministerpräsidialamtes, Fonds Maliyeden Müdevver $\mathrm{n}^{\circ} 8$. Ab und zu findet man auch den Ausdruck „kötürüm“, verkrüppelt, an den Beinen behindert, mit anderen Worten zum Dienst ungeignet (Fol. $3 \mathrm{v}^{\circ}, 57 \mathrm{v}^{\circ}$ ) und ,ga'ib“ verschollen $\left(\right.$ Fol. $\left.28 \mathrm{r}^{\circ}\right)$. 
dem Balkan in der ersten Hälfte des 16. Jahrhunderts dokumentarisch zu belegen, was nicht heißen soll, dass es vorher keine gab. Es wird auch behauptet, dass einige mosaischen Glaubens waren, wobei man sich als Erstes auf die Legende Demir Babas stützt. Da es sich hier um eine starke Auseinandersetzung zwischen den Insassen zweier Klöster handelt, kann das Wort Jude möglicherweise auch nur ein Schimpfwort gewesen sein, genau so wie im Fall des Ausdrucks türkische Schweine für die Gegenpartei. Auch von Torlak Hu Kemal, der in Westanatolien bei Manisa im Landkreis Saruhan lebte und der den oben genannten Aufstand angezettelt hat, wird berichtet, dass er ein zum Islam übergetretener Jude gewesen sei. Der in manchen Chroniken verstümmelte Zusatz zu seinem Namen, für den es vorläufig keine Erklärung gibt, kann augenblicklich mangels anderer Quellen nicht als Beweis gelten. Somit bleiben wir vorläufig im Bereich der Vermutung.

Die Torlak haben auf dem Balkan einen slawischen Dialekt gesprochen und auch aus dem oben erwähnten Steuerregister TD 382 geht hervor, dass sie slawische Namen trugen.

Die osmanischen Texte lassen zwei Kategorien von Torlak erkennen. Die einen gehörten zur Kategorie des Militärs. Der Staat gewährte ihnen ein Stück Land für ihren Lebensunterhalt. Als Gegenleistung waren sie dazu verpflichtet, Militärdienst zu leisten. Im Balkan reparierten sie Festungen und bewachten Gebirgspässe, in Kleinasien dienten sie im Falle eines Krieges als Infanteristen. Diese Kategorie von Torlak blieb unbeachtet, mit anderen Worten: wir erfahren von ihnen nur etwas aus den Steuerregistern und nicht aus literarischen Quellen. Eine Ausnahme bildet die Lebensgeschichte des Scheichs Bedreddin, wobei sich aber im Falle von Torlak Hu Kemal der Text nicht klar ausdrückt, womit der Rebelle und seine zahlreichen Kämpfer sich ihr Brot verdient haben. Den Chroniken kann man in diesem Fall nicht trauen, weil sie nur das ins Licht rücken, was ihre Verurteilung als Ketzer rechtfertigt. Der Landkreis von Manisa zählte im 16. Jahrhundert im Verhältnis zu anderen eine besonders große Anzahl von Infanteristen. ${ }^{30}$

Anders verhält es sich mit den Torlak, die Menavino beschreibt. Aufgewachsen am Hofe des Sultans, muss er in den Straßen Konstantinopels diesen müßigen Bettlern begegnet sein, die die Leichtgläubigkeit der Bevölkerung ausnützten, von Almosen lebten, Drogen zu sich nahmen und ein verwerfliches Leben führten. Ihr Aussehen und ihr skandalöses Verhalten konnte nicht unbeachtet bleiben. Sie erregten einerseits die Neugierde der Bevölkerung und andererseits Abscheu über ihre Lebensart. Ein solcher Unterschied zwischen den zwei Ka-

30 Emecen 1989; auf den Seiten 310-328, verschiedene Listen von Infanteristen aus mehreren Steuerregistern von ca. 1487 bis ca. 1582. 
tegorien lässt sich meines Erachtens nur auf das gesellschaftliche Absinken einer Schicht zurückführen, die nicht mehr im Dienste des Staates ihr Leben verdienen konnte oder wollte.

\section{b. Ursprung der Torlak}

Um eine Antwort auf diese Frage zu bekommen, sollte man drei Fakten in Betracht ziehen: die geographische Lage auf europäischem und anatolischem Boden, den Einsatz dieser Menschen für militärische Dienste und den eventuellen Ursprung des Namens.

In Europa trifft man sie, wie schon gesagt, auf dem südlichen Balkan an. In Anatolien findet man sie nur auf dem Gebiet, das in der Mitte des 13. Jahrhunderts noch byzantinisch war, mit anderen Worten an der Küste des Ägäischen Meeres, in Bithynien und im westlichen Teil von Phrygien. Eine Suche nach Torlak im restlichen Anatolien war bisher vergeblich.

Die Torlak leisteten Militärdienst im osmanischen Reich, ob in Europa oder in Anatolien. Sie waren aber keine Söldner, sondern bekamen vom Staat ein Stück Ackerland zum Anbauen. Dafür zogen sie in den Krieg oder leisteten ähnliche Dienste.

Was den Ursprung des Namens betrifft, so ist man sich darüber nicht einig. Torlak kommt in türkischen Wörterbüchern vor. Das Wort bedeutet ,jung, unerfahren, bartlos“ “. ${ }^{31}$ Man vergleiche es mit dem Wort tor, das ,ungeschickt"“ bedeutet, aber auch ein „schwer zu bändigendes Pferd“, oder ein Pferd, das kein arabisches Blut hat, also nicht reinrassig ist. ${ }^{32}$ Dieselbe Erklärung gibt Redhouse. ${ }^{33} \mathrm{Im}$ Persischen findet man laut Steingass das Wort tor (wild, terrified) und tori (fear, dread, terror, horror, savageness, wildness). ${ }^{34}$ Allerdings lässt uns der Autor über den Ursprung im Dunkeln.

Verlockend ist eine Verbindung mit der Kirche des Heiligen Thorlak herzustellen. Sie war die letzte Kirche, die in Konstantinopel für die Waräger gebaut wurde. ${ }^{35}$ Aber eine Reihe von Tatsachen sprechen dagegen. Der Heilige Thorlak war ein isländischer katholischer Bischof, so auch die skandinavische Gemeinde nach der Besetzung Konstantinopels anlässlich des IV. Kreuzzugs, und sie sprach sicher ihren nordischen Dialekt und nicht slawisch. Nach der Wiedereroberung Konstantinopels durch Michael VIII. hört man nichts mehr von dieser Kirche.

31 Tarama Sözlü̆̆̈̈, Bd.V, 1971, 3829.

32 Vergleiche Tor, ebd., 3827.

33 Redhouse 1921, 1253 (Tor), 1254 (Torlak auch im Sinne eines nicht dressierten Pferdes).

34 Steingass 1970, 821.

35 van Arkel-de Leeuw van Weenen / Ciggaar 1979. 
Ein Blick ins Internet öffnet eine neue Perspektive. Der Gott Thor hat in den nordischen Sprachen zu einer großen Anzahl von Eigennamen geführt, teils männliche, teils weibliche. Unter den männlichen Namen erscheint im Norwegischen Torlak und die weibliche Form Torlaug. ${ }^{36}$ Das erklärt auch den Namen des isländischen Bischofs, genannt der Heilige Torlak, dem in Konstantinopel eine Kirche gewidmet war.

Nach den vielen Einzelheiten, die man heute aus den Quellen schöpfen kann, besteht nun die Möglichkeit, dass eine Gruppe von Menschen aus Skandinavien unter einem Führer namens Torlak ausgewandert und im Osten Europas dem Einfluss einer orthodoxen, slawischen Bevölkerung ausgesetzt worden ist. Selbst wenn man von einem slawischen Dialekt spricht, so hat er laut Spezialisten mit der serbischen und bulgarischen Sprache nichts zu tun. Sie wurden wie die meisten Migranten aus dem hohen Norden für ihre Tapferkeit geschätzt und deshalb als Kämpfer eingesetzt. Sie haben allerdings nie hohe militärische Grade erreicht und bei der einheimischen Bevölkerung müssen sie nicht besonders geschätzt gewesen sein, was die Ausdrücke wie ,wild, furchterregend, nicht gebändigt, jung, unerfahren " bezeugen. Während sie in Anatolien schneller islamisiert wurden, blieb auf dem Balkan die Mehrheit orthodox. Der Name wurde schließlich zum Schimpfwort wie in der Legende Demir Babas und wie es manche Wörterbücher beweisen. ${ }^{37}$

Fassen wir das Gesagte in einigen Worten zusammen. Die Torlak waren eine Volksgruppe, die man bisher hauptsächlich aus dem Balkan kannte. Nach den vielen hier und da verstreuten Informationen, die inzwischen ans Licht gekommen sind, kamen sie aus den skandinavischen Ländern. Im Balkan gehörte die Mehrheit der orthodoxen Religion an, wie es die slawischen Namen in den osmanischen Steuerregistern beweisen. Ein Teil von ihnen siedelte nach Anatolien über, wobei sie die Westküste der Ägäis sowie Bithynien und einen Teil Phrygiens nicht überschritten. Es handelt sich also um eine Bewegung von Europa nach Kleinasien und nicht umgekehrt. Im Osmanischen Reich wurden sie wie im Balkan als auch in Anatolien zu militärischen Zwecken angeheuert. Sie reparierten Burgen, waren Passwächter oder Infanteristen. Als mit der Zeit die Infanteristen von den Janitscharen abgelöst wurden, wurden sie brotlos und

36 Google.fr vom 25. November 2010 unter „Der Tor, allemand, étymologie“: Am Ende der Abteilung „Thor, Archive Multilingue“ befindet sich ein Paragraph „Persönliche Namen": <http://www.worldlingo.com/ma/enwiki/fr/Thor>.

37 Popović 1895, 412: torlak= „Großsprecher, Maulheld“; Zeitwort: torlati: „,großschreien, prahlen“; Zenker, ${ }^{21972,} 607$ (paresseux, négligent, ridicule, ignorant, idiot). 
verdienten ihren Lebensunterhalt durch Wahrsagerei. Ein Teil konnte aber in der Bevölkerung untertauchen. Im Internet findet man heute türkische Bürger, die den Familiennamen Torlak tragen.

\section{Literaturverzeichnis}

Âşıkpaşaoğlu Ahmed Âşıkî, 1947: Tevârîh-i Âl-i Osman, (Edition in lateinischen Buchstaben von Çiftçioğlu N. Atsız), Istanbul.

Babinger, F., 1962: „Das Bektaschi-Kloster Demir Baba“, in: Aufsätze und Abhandlungen zur Geschichte Südosteuropas und der Levante, Bd. I, München, 88-96. (=Mitteilungen des Seminars für Geschichte und Orientalische Sprachen, Bd. XXXIV, Berlin, 1931, 1-10).

Babinger, F., 1921: „Schejch Bedr ed-din, der Sohn des Richters von Simāw, Ein Beitrag zur Geschichte des Sektenwesens im altosmanischen Reich“, in: Der Islam, Bd. XI, Berlin-Leipzig, 1-106.

Balivet, M., 1995: Islam mystique et révolution armée dans les Balkans ottomans; vie du Cheikh Bedreddin le ,Hallâj” des Turcs " (1358/59-1416). Istanbul.

Cotsonis, H. I., 1957: „Aus der Endzeit von Byzanz: Bürklüdsche Mustafa, ein Martyrer für die Koexistenz zwischen Islam und Christentum“, in: Byzantinische Zeitschrift, Bd. 50/2, München, 397-404.

370 numaralı Muhâsebe-i Vilâyet-i Rûm-Ili Defteri (937/1530), Bd. I, Ankara, 2001; Bd.II, 2002.

Ducas, 1958: Istoria Turco-Bizantina (1341-1462), (griechischer Text und Übersetzung auf Rumänisch von V. Grecu). Bukarest.

Ducas, 1975: Decline and Fall of Byzantium to the Ottoman Turks by Doukas, (englische Übersetzung von Magoulias H.J). Detroit.

Ducas, 2008: Histoire turco-byzantine, (Introduction, traduction et commentaire, ARNT [Atelier National de Reproduction des Thèses], übersetzt von Dayantis, J.) Lille.

Emecen, F. M., 1989: XVI. Astrda Manisa Kazası. Ankara.

Gölpınarlı, Abdülbâki, 1966: Simavna Kadısıoğlu Şeyh Bedreddin, (mit einer Einleitung von Ismet Sungurbey). Istanbul.

Halil bin Ismâîl bin Şeyh Bedrüddîn Mahmûd, 1967: Simavna Kadısıŏ̆lu Şeyh Bedreddin Manâkıbı, (hrsg. von Gölpinarlı, Abdülbâki / Sungurbey, Ismet). Istanbul.

Inalcık, H. Hicrî, 1954: 835 tarihli sûret-i defter-i sancak-i Arvanid. Ankara.

Karadzić, Vuk Stefanović, ${ }^{2} 1852$ : Srpski rječnik istumačen njemačkijem i latinskijem riječima; (skupio ga i na svijet izdao Vuk Stef. Karadzić, u Beču, u stampariji jermenskoga manastira), (=Lexicon serbico-germanico-latinum, edidit Vuk Steph. Karadschitsch, Vindobonae, Typis Congregationis Mechitaristicae, 1852).

Kiel, Machiel, 1991: „Hrāzgrad-Hezārgrad-Razgrad. The Vicissitudes of a TurkishTown in Bulgaria“, in: Turcica, Bd. XXI-XXIII, 495-563. 
Kissling, H. J., 1950: „Das Menāqybnāme Scheich Bedr-ed-Din's, des Sohnes des Richters von Samāvnā", in: Zeitschrift für die Kunde des Morgenlandes, Bd. 100, 1950, 112-176.

Kreutel, R. F., 1959: Vom Hirtenzelt zur Hohen Pforte, Frühzeit und Aufstieg des Osmanenreiches nach der Chronik „Denkwürdigkeiten und Zeitläufte des Hauses 'Osman“. Graz, Wien, Köln.

Mala Enciklopedija, Prosveta, Beograd, ${ }^{2} 1969$, Bd. 2.

Menavino, Giovanni, Antonio, 1548: Trattato de'costumi et vita de'turchi (<http:// books. google.fr).

Milenović, Miomir, 1929: Artikel „Torlak“, in: Narodna Enciklopedija srpsko-hrvatsko-slovenačka, Band IV (s-šs), Zagreb, 576.

Neşri, Mehmed, 1995: Kitâb-ı Cihan-Nümâ; Neşrî Tarihi, Bd. II, (ed. F.R. Unat und M.A. Köymen; osmanischer Text mit Umschrift in lateinischen Buchstaben), Ankara.

Nicolay, Nicolas de, 1989: Dans l'Empire de Soliman le Magnifique, présenté et annoté par Marie-Christine Gomez-Géraud et Stéphane Yerasimos, Paris.

Popović, G., 1895: Rečnik Srpskoga i Nemačkoga Jezika. Panćevo.

Redhouse, J.W., 1921: A Turkish and English Lexicon. Constantinople.

Soustal, P., 1991: Thrakien (= Tabula Imperii Byzantini 6.) Wien.

Spandounes, Theodore, 1997: On the Origin of the Ottoman Emperors, (übersetzt von Nicol, D.M.) Cambridge.

Spandugino, Théodore: Petit traicté de Théodore... Cantacusin [Contacuzène], Constantinopolitain, de l' Origine des princes ou empereurs des Turcz, ordre de leur court et coustumes de la nation, Manuskript n ${ }^{\circ}$ 797, Bibliothèque du Château de Chantilly, Manuskript 797. (Musée de Condé in Chantilly, alte $\mathrm{N}^{\circ}$ XIV H 97) fol. 49r ${ }^{\circ}-50^{\circ}$; in: URL: <http:// www.calames.abes.fr/pub/\#details?id=IF3011295\%23culture $=f r>$.

Steingass, F., ${ }^{2}$ 1970: Persian-English Dictionary. Beirut.

Tarama Sözlü̈̆̈̈, Bd.V, 1971, Ankara.

van Arkel-de Leeuw van Weenen, Andrea / Ciggaar, Krijnie: „St. Thorlac's in Constantinople built by a Flemish Emperor", in: Byzantion, Bd. XLIX, 1979, 428-446.

Villain-Gandossi, Christiane, 1979: „La cronaca italiana di Teodoro Spandugino“, in: Il Veltro, Jahrgang XXIII, 2-4, Rom, S. 151-172.

Yıldırım, Riza, 2010: „Muhabetten Tarikata - Bektaşi Tarikatı'nın Oluşum Sürecinde Kızıldeli'nin Rolü“, in: Türk Kültürü ve Hacı Bektaş Veli Araştırma Dergisi, Bd. 53(2010), Ankara, 153-190.

Zenker, J. Th., ${ }^{21972: ~ T u ̈ r k i s c h-a r a b i s c h-p e r s i s c h e s ~ H a n d w o ̈ r t e r b u c h . ~ H i l d e s h e i m, ~ N e w-Y o r k . ~}$ 
